\title{
APPROXIMATION TO IRRATIONALS BY CLASSES OF RATIONAL NUMBERS
}

\section{LEONARD TORNHEIM}

Hurwitz [3] proved that there exist infinitely many rational numbers $a / b$ for every irrational $\xi$ such that

$$
|\xi-a / b|<k / b^{2}
$$

if and only if $k \geqq 1 / 5^{1 / 2}$. Scott [11] proved that if the fractions $a / b$ are restricted to any one of the three classes (i) $a, b$ both odd, (ii) $a$ even, $b$ odd, or (iii) $a$ odd, $b$ even, the same conclusion holds if $k \geqq 1$. Other proofs of this have been given by Oppenheim [8], Robinson [10], and Kuipers and Meulenbeld [6]. Robinson also showed that if any pair of these classes were used, then $k \geqq 1 / 2$.

Let $(m, r, s)=1$; then the set of all fractions $a / b$ in lowest terms for which $a \equiv r, b \equiv s(\bmod m)$ will be denoted by $\langle r, s\rangle$. Descombes and Poitou $[1 ; 9]$ have investigated the values of $k$ needed for sets $\langle r, s\rangle$. Hartman [2] and Koksma [5] have considered the problem of a universal constant for all sets of fractions $a / b$ with $a \equiv r, b \equiv s(\bmod m)$ where $a, b$ need not be relatively prime nor is it required that $(r, s, m)$ $=1$.

We obtain results for other classes of rational numbers.

Let the continued fraction expansion of $\xi$ be $\xi=\left[d_{0}, d_{1}, \cdots\right]$; then the $n$th convergent is $a_{n} / b_{n}=\left[d_{0}, d_{1}, \cdots, d_{n}\right]$ and the $n$th denominator is $d_{n}$. We shall use the following known results $[4 ; 8 ; 10]$.

LemmA A. If $a_{n-1} / b_{n-1}, a_{n} / b_{n}, a_{n+1} / b_{n+1}$ are three consecutive convergents to $\xi$, then at least one of them satisfies (1) with $k=1 / 5^{1 / 2}$.

Lemma B. Let $a_{n} / b_{n}, a_{n+1} / b_{n+1}$ be two consecutive convergents to $\xi$. Then at least one of them satisfies (1) with $k=1 / 2$. The same is true with $k=1$ of one of $\left(a_{n+1}-a_{n}\right) /\left(b_{n+1}-b_{n}\right)$ and $\left(a_{n+1}+a_{n}\right) /\left(b_{n+1}+b_{n}\right)$.

Lemma C. If $(a, b)=1$ and if $|\xi-a / b|<1 / b^{2}$, then $a / b$ is either $a_{n} / b_{n},\left(a_{n}+a_{n+1}\right) /\left(b_{n}+b_{n+1}\right)$, or $\left(a_{n}-a_{n-1}\right) /\left(b_{n}-b_{n-1}\right)$ for a suitable $n$.

Lemma D. If $a_{n-1} / b_{n-1}, a_{n} / b_{n}, a_{n+1} / b_{n+1}$ are three consecutive convergents, then $a_{n+1}=d_{n+1} a_{n}+a_{n-1}, b_{n+1}=d_{n+1} b_{n}+b_{n-1}$, where $d_{n}$ is the nth denominator, and $a_{n} b_{n+1}-a_{n+1} b_{n}= \pm 1$.

If $|\xi-a / b|=c / b^{2}$, we call $c$ the approximation coefficient of $a / b$ (for $\xi$ ).

Presented to the Society, May 1, 1954; received by the editors May 20, 1954. 
LEMMA E. The approximation coefficient $k_{n}$ of the nth convergent $a_{n} / b_{n}$ of $\xi$ is given by $1 / k_{n}=\left[d_{n+1}, d_{n+2}, \cdots\right]+1 /\left[d_{n}, d_{n-1}, \cdots, d_{1}\right]$.

Let $C$ be a certain class of fractions $a / b$. We shall say that $k_{0}$ is the approximation coefficient for the class $C$ if it is true that for every irrational $\xi$ there exist infinitely many rational numbers $a / b$ in $C$ such that (1) holds if and only if $k \geqq k_{0}$.

Lemma 1. Let $m=p^{e}$ ( $p$ an odd prime). Then the approximation coefficient of the class of rational numbers $a / b$ with $(a, m)=1$ is $1 / 5^{1 / 2}$.

Suppose that $\left(a_{n}, p\right)=p$. Therefore $\left(a_{n+1}, p\right)=1$ by Lemma $\mathrm{D}$. Next $a_{n+2}=a_{n}+d_{n+2} a_{n+1}$. If $d_{n+2} \geqq 3, a_{n+1} / b_{n+1}$ is satisfactory by Lemma E. But if $d_{n+2}$ is 1 or 2 , then $\left(d_{n+2}, p\right)=1$ since $p$ divides $a_{n}$ and not $d_{n+2} a_{n+1}$. If $d_{n+3} \geqq 3$, then as before we see that $a_{n+2} / b_{n+2}$ is satisfactory. Now suppose $d_{n+2}$ and $d_{n+3}$ are both less than 3 and one of them is 2 ; if $d_{n+2}=2$, then $a_{n+1} / b_{n+1}$ is satisfactory for by Lemma E, $1 / k_{n+1} \leqq[2,2,1, \cdots]+[0, \cdots]>7 / 3>5^{1 / 2}$; if $d_{n+3}=2$ then similarly $a_{n+2} / b_{n+2}$ will do. Otherwise $a_{n+1} / b_{n+1}, a_{n+2} / b_{n+2}$, $a_{n+3} / b_{n+3}$ are three consecutive convergents with numerators prime to $p$. By Lemma $A$, at least one is satisfactory.

We have shown that if $\left(a_{n}, p\right)=p$, at least one of the three following convergents is effective. Now either $\left(a_{n}, p\right)=p$, or $\left(a_{n+1}, p\right)=p$, or $\left(a_{n+2}, p\right)=p$, or $\left(a_{n} a_{n+1} a_{n+2}, p\right)=1$. In this last case one of $a_{n} / b_{n}$, $a_{n+1} / b_{n+1}, a_{n+2} / b_{n+2}$ will do. Thus we see that in all cases among any six consecutive convergents of $\xi$ at least one is of the type described in the lemma.

That the constant $1 / 5^{1 / 2}$ cannot be decreased follows from the known fact that an irrational $\xi$ requires this value if and only if its denominators are ultimately all 1 . The set of such numbers is denumerable.

Lemma 2. Let $m=2^{\circ}(e \geqq 1)$. Then the approximation coefficient of the class of rational numbers $a / b$ with a prime to $m$ is $1 / 2$.

This result was proved by Robinson [10].

Lemma 3. Let $m=p^{e} q^{f}$, where $p, q$ are distinct primes and $e, f$ are both positive. Then the approximation coefficient of the class of all rational numbers $a / b$ with $(a, m)=1$ is 1 .

Let $a_{n} / b_{n}, a_{n+1} / b_{n+1}$ be two consecutive convergents of $\xi$. If either has numerator prime to $m$, then that fraction approximates sufficiently closely by Lemma E. Otherwise $a_{n}$ is divisible by either $p$ or $q$, say $p$, and $a_{n+1}$ is divisible by $q$ since $\left(a_{n}, a_{n+1}\right)=1$ by Lemma D. 
But then $a_{n+1} \pm a_{n}$ are prime to $m$ and one of $\left(a_{n+1} \pm a_{n}\right) /\left(b_{n+1} \pm b_{n}\right)$ approximates $\xi$ sufficiently closely by Lemma B.

To prove the converse, let $\xi=\left[0, p^{e}, w, z_{3} m, z_{4} m, \cdots\right]$ where $w$ is chosen so that $w p^{f}+1 \equiv 0\left(\bmod q^{s}\right)$ and $z_{n} \rightarrow \infty$. Then using Lemma D we see that no convergent has numerator prime to $m$. Any $a / b$ satisfying (1) with $k=1$ is thus one of $\left(a_{n} \pm a_{n+1}\right) /\left(b_{n} \pm b_{n+1}\right)$ for some $n$ by Lemma D. But if $\alpha_{n}=\left[d_{n}, d_{n-1}, \cdots, d_{1}\right], \beta_{n}=\left[d_{n+1}, d_{n+2}, \cdots\right]$, then $\left(a_{n}+a_{n+1}\right) /\left(b_{n}+b_{n+1}\right)$ has approximating coefficient $1 /\{1$ $\left.+1 /\left(\beta_{n}-1\right)+1 /\left(\alpha_{n}+1\right)\right\}$ and $\left(a_{n+1}-a_{n}\right) /\left(b_{n+1}-b_{n}\right)$ has approximating coefficient $1 /\left\{1+1 /\left(\alpha_{n+1}-1\right)-1 /\left(\beta_{n+1}+1\right)\right\}$. Since $\alpha_{n} \rightarrow \infty, \beta_{n}$ $\rightarrow \infty$, the theorem follows. The set of such numbers $\xi$ has the power of the continuum.

Lemma 4. For every pair of classes $\langle r, s\rangle$ and $\left\langle r^{\prime}, s^{\prime}\right\rangle$ there is a unimodular linear fractional transformation

$$
z^{\prime}=(A z+B) /(C z+D)
$$

where $A, B, C, D$ are integers and $|A D-B C|=1$, such that the class $\langle r, s\rangle$ is sent onto the class $\left\langle r^{\prime}, s^{\prime}\right\rangle$.

It is an elementary result that there exists a transformation which sends a given fraction $a / b$ into a given $a^{\prime} / b^{\prime}$. It is easy to see that any other fraction in $\langle a, b\rangle$ will have its image in $\left\langle a^{\prime}, b^{\prime}\right\rangle$. The inverse transformation shows that the mapping is onto.

LEMMA 5. The approximation coefficients of the classes $\langle r, s\rangle$ are all the same for a given $m$.

This result was stated by Descombes and Poitou [1]. We require the following proof in order to prove the next lemma.

Let $k$ be the approximation coefficient of a class $\langle r, s\rangle$. Then

$$
k=\sup _{\xi}\left(\liminf _{a / b} b^{2}|\xi-a / b|\right)
$$

where $a / b$ ranges in the class $\langle r, s\rangle$. We shall show that the same value of $k$ is obtained if $a / b$ ranges through the class $\left\langle r^{\prime}, s^{\prime}\right\rangle$.

For if $\xi^{\prime}, a^{\prime} / b^{\prime}$ are the images of $\xi, a / b$ and if $b^{2}|\xi-a / b|=k_{0}$, then

$$
b^{\prime 2}\left|\xi^{\prime}-a^{\prime} / b^{\prime}\right|=k_{0}^{\prime}=k\left[\frac{C a^{\prime} / b^{\prime}+D}{C a^{\prime} / b^{\prime}+D+C k / b^{2}}\right] .
$$

As $a / b \rightarrow \xi, q \rightarrow \infty$, and $k_{0}^{\prime} \rightarrow k_{0}$. Hence

$$
\lim \inf k_{0}^{\prime}=\lim \inf k_{0}
$$

and the lemma follows. 
The set of all classes $\langle r t, s t\rangle$ for fixed $r, s$ and for all $t$ prime to $m$ will be called the class $\{r, s\}$. If a transformation (2) sends $\langle r, s\rangle$ onto $\left\langle r^{\prime}, s^{\prime}\right\rangle$, it sends $\langle r t, s t\rangle$ onto $\left\langle r^{\prime} t, s^{\prime} t\right\rangle$. Hence the class $\{r, s\}$ is sent onto $\left\{r^{\prime}, s^{\prime}\right\}$. This implies the next lemma.

LeMma 6. The approximation coefficients of the classes $\{r, s\}$ are all the same for a given $m$.

Theorem 7. Let $(r, s, m)=1$. Then the approximation coefficient of the class of all rational numbers $a / b$ with $a \equiv r t, b \equiv s t(\bmod m)$, where $t$ is an integer depending on $a, b$, is $m / 5^{1 / 2}$.

Robinson [10] proved this result for the case $m=2$.

We prove the theorem for the case $s=0$. The proof in general then follows from an argument similar to that used in the proof of Lemma 6. Thus $b=d m$. The rational approximations $a / d m$ to $\xi$ are in one-toone correspondence with the rational approximations $a / d$ to $m \xi$. Since $|a / d-m \xi|>k / d^{2}$ is satisfied for infinitely many $a / d$ for each irrational $\xi$ if and only if $k \geqq 1 / 5^{1 / 2}[2]$, we see that $|a / d m-\xi|<k m /(d m)^{2}$ holds for infinitely many $a / d m$ if and only if $k \geqq 1 / 5^{1 / 2}$.

Theorem 8. Let $(r, s, m)=1$. The approximation coefficient of the class of all rational numbers $a / b$ in lowest terms with $a \equiv r t, b \equiv s t(\bmod m)$ where $t$ is an integer depending on $a, b$, is:

$m / 5^{1 / 2}$ if $m=p^{e}, p$ an odd prime;

$m / 2$ if $m=2^{e}, e \geqq 1$;

$m$ if $m=p^{\circ} q^{f}, p$ and $q$ distinct primes, $e$ and $f$ positive.

By Lemma 6 it suffices to prove the theorem when $s=0$. A discussion similar to that in the proof of the preceding theorem shows that this theorem follows from Lemmas 1, 2, and 3.

Our final result is a generalization of Lemmas 1,2 , and 3 .

Theorem 9. Let $m=u v$ where $(u, v)=1$. Then the set of rational numbers $a / b$ in lowest terms such that $(a, v)=1$ and $(b, u)=1$ has $a p$ proximation coefficient

$1 / 5^{1 / 2}$ if $m=p^{e}, p$ an odd prime;

$1 / 2$ if $m=2^{e}, e \geqq 1$;

1 if $m=p^{e} q^{f}, p$ and $q$ distinct primes, $e$ and $f$ positive.

The proof reverses the argument used to obtain Theorem 8 from Lemmas 1, 2, and 3. Let $r=v, s=u$. Then the class $\{r, s\}$ has approximation coefficient $\mathrm{km}$ as given in Theorem 8 . All fractions $a^{\prime} / b^{\prime}$ in $\{r, s\}$ have $a^{\prime}=a v$ and $b^{\prime}=b u$, where $a, b$ are integral. Hence $|\xi-a v / b u| \leqq k m /(b u)^{2}$ and therefore $|(u / v) \xi-a / b|<(u / v) k u v /(b u)^{2}$ $=k / b^{2}$. 


\section{BiBLIOGRAPHY}

1. R. Descombes and G. Poitou, Sur certains problèmes d'approximation, C. R. Acad. Sci. Paris vol. 234 (1952) pp. 581-583.

2. S. Hartman, Sur une condition supplémentaire dans les approximations diophantiques, Colloquium Mathematicum vol. 2 (1949) pp. 48-51.

3. A. Hurwitz, Über die angenäherte Darstellung der Irrationalzahlen durch Brüche, Math. Ann. vol. 39 (1891) pp. 279-284.

4. J. F. Koksma, Diophantische Approximationen, 1936.

5. - Sur lapproximation des nombres irrationnels sous une condition supplementaire, Simon Stevin vol. 28 (1951) pp. 199-202.

6. L. Kuipers and B. Meulenbeld, Some properties of continued fractions, Acta Math. vol. 87 (1951) pp. 1-12.

7. - On a certain classification of the convergents of a continued fraction I, Nieuw Archief voor Wiskunde vol. 3 (1953) pp. 199-211.

8. A. Oppenheim, Rational approximation to irrationals, Bull. Amer. Math. Soc. vol. 47 (1941) pp. 602-604.

9. G. Poitou and R. Descombes, Sur certains problèmes d'approximation. II. C. R. Acad. Sci. Paris vol. 234 (1952) pp. 1522-1524.

10. R. M. Robinson, The approximation of irrational numbers by fractions with odd or even terms, Duke Math. J. vol. 7 (1940) pp. 354-359.

11. W. T. Scott, Approximation to real irrationals by certain classes of rational fractions, Bull. Amer. Math. Soc. vol. 46 (1940) pp. 124-129.

UNIVERSITY OF MichigaN 